CASE REPORT

\title{
Bullous eyelid
}

\author{
Zahra N Jaffer, ${ }^{1,2}$ Cherie Nicholson ${ }^{3}$
}

${ }^{1}$ Department of Family and Community Medicine, Faculty of Medicine, University of Toronto, Toronto, Canada

${ }^{2}$ Family Medicine, Weeneebayko Area Health Authority, Moose Factory, Canada

${ }^{3}$ Postgraduate Medicine, University of Toronto, Toronto, Canada

\section{Correspondence to} Dr Zahra N Jaffer, zahra.jaffer@waha.ca

Accepted 19 January 2018
Check for updates

To cite: Jaffer ZN Nicholson C. BMJ Case Rep Published Online First: [please include Day Month Year]. doi:10.1136/bcr-2017220962

\section{SUMMARY}

Periocular necrotising fasciitis is a rare but sightthreatening condition, which relies on clinical judgement to detect in a timely manner. A 51-year-old woman presented to a rural hospital with rapid onset bilateral eye swelling, erythema and pain and was started on broad spectrum intravenous antibiotics. Upon admission, she became septic and required fluid resuscitation and transfer to a higher level of care. She received debridement and continued intravenous antibiotics, with step down to oral when clinically stable. Rapid recognition and treatment of her condition resulted in a positive outcome.

\section{BACKGROUND}

Periocular necrotising fasciitis (PONF) is a rare, rapidly spreading life and sight-threatening condition. Mortality is related to a delay in diagnosis, usually stemming from a lack of awareness of the disease. For General Practitioners working in a remote, low-resource setting, it is important to recognise the condition in order to ensure timely access to further specialist management.

We present a case of PONF in a 51-year-old woman where rapid recognition, initiation of treatment and transfer to a higher level of care resulted in a positive outcome.

\section{CASE PRESENTATION}

A 51-year-old woman presented to the emergency department (ED) of a remote town with a 12-hour history of worsening bilateral periorbital swelling. She had a medical history of systemic lupus erythematosus (SLE) and Lynch syndrome. Her medications included hydroxychloroquine, folic acid, iron, vitamin B12 and acetaminophen as needed. She noticed mild swelling of the right upper eyelid the previous night and the next morning found both eyes swollen and was unable to open her left eye. She denied any recent illnesses, trauma or medication changes.

On arrival to the ED, she was alert with normal mentation, blood pressure $116 / 70 \mathrm{~mm} \mathrm{Hg}$, heart rate 102 , respiratory rate 20 , temperature $39.9^{\circ} \mathrm{C}$ and oxygen saturation $96 \%$ on room air. On examination, she had bilateral periorbital and infraorbital swelling and an erythematous left eye surrounded by pustules with overlying bullae. Extensive upper and lower eyelid swelling and tenderness precluded left eye examination. The right eye had mild swelling of the upper and lower eyelid, diffuse erythema and minimal tenderness with purulent discharge at the medial and lateral corners. The remainder of the eye examination was normal; visual acuity could not be assessed. The exudate was swabbed for culture and the patient was started on piperacillintazobactam $4.5 \mathrm{~g}$ intravenously every 8 hours and vancomycin $1500 \mathrm{mg}$ intravenously every 12 hours. She was admitted to our team with a diagnosis of preseptal cellulitis.

On taking over care, we noted her vital signs had worsened with a temperature of $40.5^{\circ} \mathrm{C}$, heart rate 91 and blood pressure 98/59 mm Hg. Her leucocyte count was 18.8 (normal 4.0-10.5) $\times 10^{9} / \mathrm{L}$.

On re-examination, one of the bullae over her left eyelid had ruptured, leaking serous fluid. She was extremely tender to touch with worsening bilateral orbital and infraorbital swelling. Violaceous skin changes were noted on the left medial canthal area (figure 1). Fluid resuscitation was commenced with normal saline.

Ophthalmology was consulted via phone due to the possibility of orbital cellulitis; they requested visualisation of the left globe, which was achieved manually with intravenous analgaesia. The left conjunctiva was diffusely chemotic and erythematous. Photos of the skin changes were sent to Ophthalmology, and after discussion of the clinical findings including rapid progression, clinical deterioration and violaceous skin changes, the diagnosis of PONF was considered (figure 2).

Ophthalmology accepted transfer of the patient to a tertiary care hospital approximately 2 hours flight away. While awaiting transfer, despite $5 \mathrm{~L}$ of intravenous fluid resuscitation, her blood pressure dropped to $74 / 57 \mathrm{~mm} \mathrm{Hg}$. Peripheral norepinephrine was started at $8 \mathrm{mcg} / \mathrm{min}$ to maintain a mean arterial pressure greater than or equal to 65 . The intensivist in the Intensive care unit (ICU) was

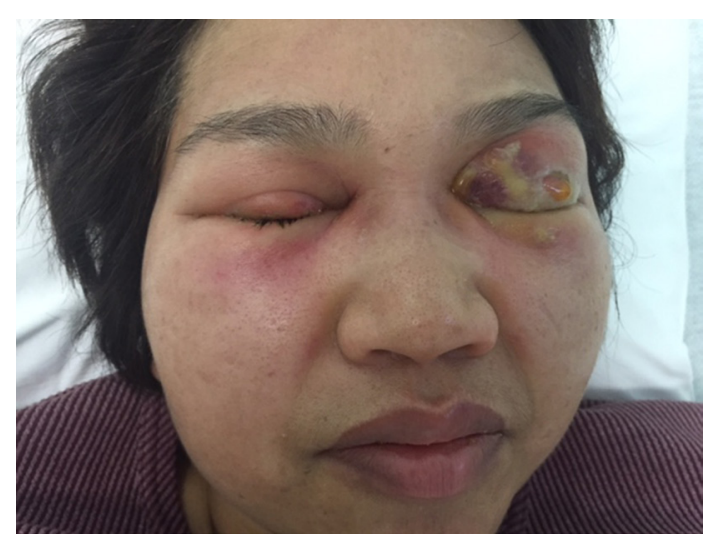

Figure 1 Left eye on presentation to inpatient department: ruptured bulla, violaceous skin changes at inner canthal area, purulence and swelling. 


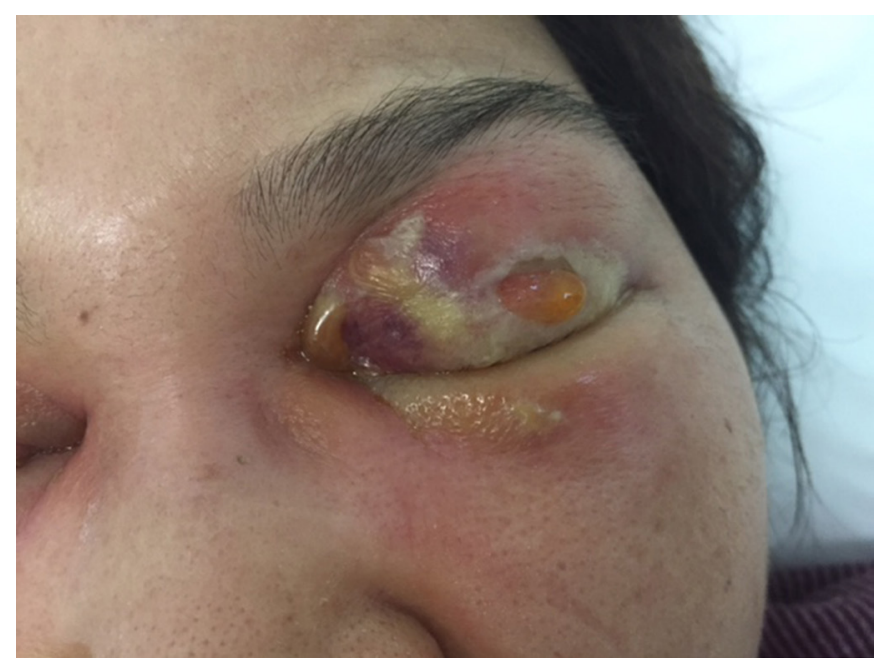

Figure 2 Close up of left eye.

consulted prior to transfer and recommended the addition of clindamycin $900 \mathrm{mg}$ intravenously for Group A Streptococcal coverage along with intravenous immunoglobulin (IVIG). Due to time constraints and limited resources, only clindamycin was administered. The patient was air-transferred approximately $800 \mathrm{~km}$ away to a step-down ICU for more comprehensive care, about 12 hours postpresentation to the ED.

\section{INVESTIGATIONS}

Initial blood and eyelid discharge swab cultures showed no growth. Tissue cultures, done at the tertiary centre, grew Group A Streptococcus (Streptococcus pyogenes). Fungal cultures were negative. Necrotic tissue sent for pathology revealed suppurative acute inflammation and gram positive cocci. CT scan showed extensive bilateral preseptal cellulitis and a large left preconjunctival abscess with no intraorbital cellulitis or abscess.

\section{DIFFERENTIAL DIAGNOSIS}

Periorbital necrotising fasciitis is a rare diagnosis. Other relevant diagnoses to consider are as follows:

- preseptal cellulitis;

- orbital cellulitis.

It is important to differentiate these diagnoses, as rapidity of treatment and consultation with specialists is important with necrotising fasciitis and orbital cellulitis.

\section{OUTCOME AND FOLLOW-UP}

The patient required debridement of her eyelid by oculoplastic surgery, where necrotic tissue of the eyelid was noted removed. She received 5 days of intravenous piperacillin-tazobactam and was stepped down to oral cefprozil on day 6 for a 14-day course along with topical erythromycin ointment for 3 weeks. Healing granulation tissue was seen soon after debridement. Her vision remained stable. She was discharged home on antibiotic therapy with oculoplastic follow-up in her home community.

\section{DISCUSSION}

Necrotising fasciitis (NF) is a rare, rapidly progressing bacterial infection involving subcutaneous soft tissues and is more commonly found to involve the abdominal wall, extremities and groin. ${ }^{12} \mathrm{NF}$ is rarely found in the periocular area with 94 documented cases over the last 50 years. ${ }^{3}$ It is more common in men, with trauma or injury as common triggers. ${ }^{3}$
Pathogenesis is multifactorial: endotoxins and exotoxins cause tissue ischaemia and systemic release of cytokines. ${ }^{1}$ Risk factors include alcoholism, diabetes mellitus, rheumatological disease, systemic malignancy and systemic corticosteroid or immunosuppressant use-though many patients are healthy with no comorbidities. ${ }^{3-6}$ Our patient with a diagnosis of SLE fit this criteria; one review of 58 cases noted $7 \%$ had rheumatological diseases. ${ }^{3}$

PONF presents uniquely in the eyelid: the thin skin and abundant blood supply of the orbicularis oculi muscle allows for earlier recognition of infection. Furthermore, the muscle, which is superficial to the orbital septum fascia, acts as a vascular barrier between the skin and spreading infection. ${ }^{57}$ The inflammation is also contained by the firm attachment of the dermis medially and laterally to the nasojugal and malar folds, limiting lateral spread. ${ }^{2}$ As the thin subcutaneous tissue over the nose has little resistance to the spread of infection, necrotising fasciitis may involve both eyes. ${ }^{5}$ Just over one-third of reported cases in the literature noted bilateral involvement. ${ }^{2}$

PONF has a mortality rate between $3 \%$ and $10 \%$ compared with $>20 \%$ for NF affecting other areas of the body. ${ }^{2-4}$ There are likely several reasons for its better prognosis compared with NF elsewhere including: (1) earlier presentation as the infection occurs in a visually obvious area, (2) better antibiotic penetration given the rich blood supply and (3) the orbital septum integrity appears to slow the posterior spread of infection with a preference for lateral spread. ${ }^{2} 7$ Though mortality is lower, PONF is associated with significant morbidity-loss of vision via central retinal artery occlusion or orbital spread, as well as tissue defects causing functional and cosmetic damage. ${ }^{2-4}$

As the global burden of chronic disease increases, high-risk rural populations such as our First Nations community are presenting with increasingly complex issues which will require rapid recognition and management by General Practitioners working in these settings. This presents a unique challenge due to the rarity of this disease-the UK incidence is estimated at 0.24 cases per million per annum. ${ }^{3}$ Our patient was initially diagnosed with preseptal cellulitis, with the severity of her condition not noted in the ED.

Distinguishing necrotising fasciitis from severe cellulitis is difficult but important: rapid identification and treatment are directly related to decreased mortality. ${ }^{14}$ Rajak et al suggest early tissue biopsy in diagnostic uncertainty; however, this is often not feasible in a rural setting. ${ }^{3}$ Thus, the clinical findings are important-interestingly, Amrith et al in a review of 94 cases noted there were minimal details in case reports explaining on what basis the actual diagnosis of NF was made. ${ }^{2}$ Lazzeri et al suggest the definition of NF as comprising three specific criteria: 'extensive necrosis of the superficial fascia, rapid spread to involve the surrounding tissue and systemic toxicity'. 6 The most common symptoms include erythema, severe tenderness and rapid periorbital swelling, which can also be present in preseptal cellulitis. Typical NF features include violaceous skin changes and bullae. ${ }^{6}$ Our patient demonstrated all of these features including all three points of the Lazzeri et al's definition, leading us to consider a diagnosis of PONF. ${ }^{6}$ Systemic symptoms and gangrenous skin changes along with loss of sensation from nerve destruction, crepitus, blindness, meningitis and multiorgan failure are later findings. ${ }^{26}$

Systemic symptoms are important in recognising the onset of sepsis; one study of 29 patients noted $86 \%$ were infected with Group B haemolytic Streptococci, and another literature review noted toxic shock in $30.9 \%$ of cases. ${ }^{23}$ Imaging such as CT or MRI is an option at centres with these modalities. ${ }^{2}$ 
Some cases may be managed conservatively with antibiotics alone: if systemically well, some advocate close clinical monitoring with inflammatory markers and continuation of medical management if all continues to improve. ${ }^{3}$ However, Amrith et al noted that all current studies are retrospective; hence, it is difficult to conclude which types of cases could be managed conservatively versus surgically. ${ }^{3}$

A literature review of 94 cases revealed $85 \%$ underwent surgical debridement-which decreases the bacterial load and hyaluronic acid production, which plays a role in infection spread. ${ }^{2}$ Furthermore, thrombosis of blood vessels due to perivascular involvement and hypercoagulability can reduce the antibiotic's reach to the infected area, making debridement an important step. ${ }^{2}$

Antibiotic choice is important: systemic broad-spectrum antimicrobial therapy consisting of a $\beta$-lactam antibiotic and clindamycin is a recommended regimen. ${ }^{2}{ }^{8}$ Clindamycin is an important addition which the specialist suggested to us, as it decreases construction of bacterial defence proteins (streptococcal $\mathrm{M}$ proteins and pyrogenic exotoxins) even at subinhibitory concentrations, is not dependent on stage of bacterial growth and demonstrates a longer postantibiotic effect than $\beta$-lactams. However, it should not be used alone, as some strains of group A streptococcus are resistant to clindamycin. ${ }^{245}$ Our area has a high burden of methicillin-resistant Staphylococcus aureus; thus, vancomycin was added.

IVIG aids in antibiotic perfusion by neutralising superantigens; while this was available at our site it was not given due to time constraints during transfer. ${ }^{2}$ Other specialist therapies such

\section{Learning points}

- Typical features to help differentiate periorbital necrotising fasciitis from preseptal cellulitis include violaceous skin changes and bullae.

- Ensure appropriate antibiotic choice, with attention to local antimicrobial resistance patterns and consideration of intravenous immunoglobulin after consultation with a specialist.

- Timely recognition of periocular necrotising fasciitis and rapid referral to an appropriate facility with surgical facilities is important to reduce mortality and morbidity. as hyperbaric oxygen are beyond the scope of this paper as they are not accessible in our remote location. ${ }^{2}$

In a rural setting, we would advocate early transfer for specialist care rather than trial of conservative management due to the difficulties faced when obtaining urgent transfers (including weather, distance, lack of access to specialist services, testing and imaging and rapid clinical deterioration). Furthermore, the critical component to reduce morbidity and mortality is early recognition and treatment including surgical debridement: one series noted $<3$ days from onset of symptoms to treatment; we were able to begin treatment and transfer our patient within 12 hours. ${ }^{5}$

PONF remains a rare case presentation that requires rapid assessment and treatment to reduce morbidity and mortality. Management in a rural or remote setting requires prompt recognition of the condition and appropriate treatment of associated septic shock, along with rapid referral and transfer to a higher level of care. Though mortality has improved, morbidity and loss of vision remain considerable risks. ${ }^{3}$

Contributors ZNJ revised the draft for critical content and completed the final version for submission. CN drafted the initial article and revised the content.

Competing interests None declared.

\section{Patient consent Obtained.}

Provenance and peer review Not commissioned; externally peer reviewed.

(C) BMJ Publishing Group Ltd (unless otherwise stated in the text of the article) 2018. All rights reserved. No commercial use is permitted unless otherwise expressly granted.

\section{REFERENCES}

1 Sarani B, Strong M, Pascual J, et al. Necrotizing fasciitis: current concepts and review of the literature. J Am Coll Surg 2009;208:279-88.

2 Amrith S, Hosdurga Pai V, Ling WW. Periorbital necrotizing fasciitis - a review. Acta Ophthalmol 2013;91:596-603.

3 Rajak SN, Figueira EC, Haridas AS, et al. Periocular necrotising fasciitis: a multicentre case series. Br J Ophthalmol 2016;100:1517-20.

4 Edlich RF, Cross CL, Dahlstrom JJ, et al. Modern concepts of the diagnosis and treatment of necrotizing fasciitis. J Emerg Med 2010;39:261-5.

5 Elner VM, Demirci H, Nerad JA, et al. Periocular necrotizing fasciitis with visual loss pathogenesis and treatment. Ophthalmology 2006;113:2338-45.

6 Lazzeri D, Lazzeri S, Figus M, et al. Periorbital necrotising fasciitis. Br J Ophthalmol 2010;94:1577-85.

7 Mehta R, Kumar A, Crock C, et al. Medical management of periorbital necrotising fasciitis. Orbit 2013;32:253-5.

8 Shah AN, Day AC, Healy VC, et al. Eyelid necrotizing fasciitis: what were the early signs? J Emerg Med 2013;44:349-51.

Copyright 2018 BMJ Publishing Group. All rights reserved. For permission to reuse any of this content visit

http://group.bmj.com/group/rights-licensing/permissions.

BMJ Case Report Fellows may re-use this article for personal use and teaching without any further permission.

Become a Fellow of BMJ Case Reports today and you can:

- Submit as many cases as you like

- Enjoy fast sympathetic peer review and rapid publication of accepted articles

- Access all the published articles

Re-use any of the published material for personal use and teaching without further permission

For information on Institutional Fellowships contact consortiasales@bmjgroup.com

Visit casereports.bmj.com for more articles like this and to become a Fellow 